\title{
Electronegativity and Mendeleev number: redefinition of two important atomic chemical descriptors
}

\author{
Artem R. Oganov \\ Skolkovo Institute of Science and Technology, 3 Nobel St., 121205 Moscow, Russia \\ a.oganov@skoltech.ru
}

The critical temperature Tc of superconductivity was recently found [1] to have a clear relationship to the Periodic Table - the highest-Tc superconducting hydrides are formed by elements forming a "belt of superconductivity", roughly between II and III groups. These elements turn out to be neighbors in the sequence of Mendeleev numbers [2], which were introduced in 1984 by Pettifor [2] and shown to approximately characterize all elements by just one number. The physical meaning of these numbers remained unclear. We have shown [3] that Mendeleev number can be obtained by principal components analysis (PCA) or even a simple linear correlation applied to the set of points in the spac "atomic radius - electronegativity - polarizability" of all elements. This dimensionality reduction gives a single variable giving mathematically the best one-parameter description of the chemistry of the elements - the Mendeleev number. We have also shown [3] that thus defined Mendeleev numbers perform better than those proposed by Pettifor [1]. Reduced-dimensionality representations (such as representation by Mendeleev numbers) allow easier visualization of big data.

Among the chemical properties of the elements, electronegativity plays perhaps the most important role - chemical reactivity of the elements, bond energies, directions and heats of reactions, and many properties of molecules and solids are related to electronegativities of the elements. The oldest and the most widely used is Pauling's scale of electronegativity, developed in 1932 (see [4]) and based on bond energies. However, later it was found (e.g., [5]) that Pauling's formula, relating bond energies with electronegativity differences, is very inaccurate for significantly ionic bonds. We have proposed [6] another formula, which works well for bonds with any degree of ionicity, and obtained a new thermochemical scale of electronegativities for all elements. New electronegativities better follow chemical intuition than traditional Pauling's values (e.g. charge transfer in transition metal borides and hydrides is described qualitatively better, and so are oxyacids).

[1] Semenok D.V., Kruglov I.A., Savkin I.A., Kvashnin A.G., Oganov A.R. (2020). On distributions of superconductivity in metal hydrides. Curr. Opin. Solid State \& Mater. Sci. 24, 100808.

[2] Pettifor D.G. (1984). A chemical scale for crystal structure maps. Solid State Commun. 51, 31-34.

[3] Allahyari Z., Oganov A.R. (2020). Nonempirical definition of Mendeleev numbers: organizing the chemical space. J. Phys. Chem. C124, 2386723878

[4] Pauling, L. The Nature of the Chemical Bond 3rd edn (Cornell University Press, 1960).

[5] Matcha, R. L. (1983). Theory of the chemical bond. 6. Accurate relationship between bond energies and electronegativity differences. J. Am. Chem. Soc. 105, 4859-4862.

[6] Tantardini C., Oganov A.R. (2021). Thermochemical electronegativities of the elements. Nature Communications 12, 2087.

Keywords: Electronegativity, atomic, chemical, Mendeleev number

This work is funded by Russian Science Foundation (grant 19-72-30043). 